\title{
LA TEORÍA Y SU FUNCIÓN EN LOS TRES MODELOS DE INVESTIGACIÓN
}

Palabras Clave: Teoría, ciencia, investigación empírico analítico, histórico hermenéutico, crítico social.

\section{- SOBRE LAS FORMAS DEL CONOCIMIENTO HUMANO Y EL CONCEPTO DE TEORÍA}

El conocimiento científico, resultado de un proceso sistemático de investigación, constituye una particular forma del conocimiento (yuxtapuesto al filosófico, al teológico y al estético) adoptada por la cultura occidental a partir del siglo XVI. La adopción de esta forma particular del conocimiento hay que comprenderla en el contexto de una tradición que viene desde la antigüedad clásica y que pasa por el medioevo, esto es, en el momento de su separación de la filosofía como saber holístico de la realidad social, entendida ésta en sus tres dimensiones clásicas: lo natural, lo humano y lo religioso.

En efecto, antes del Renacimiento,(2) los pensadores medievales y antiguos asumieron lo filosófico como un procedimiento gnoseológico natural que tenía por propósito dotar de sentido a la existencia humana a la luz de la resolución de cinco grandes preguntas: ¿Qué es
1.Trabajo presentado en el seminario de Investigación a la docente Doctora Sara Victoria Alvarado. Maestría en Educación y desarrollo $\mathrm{Hu}$ a no. CindeUniversidad de Manizales. Junio 18 de 2004.

2.Con excepción del proyecto de Alejandro en el Siglo III a.n.e. relacionado con la creación y operación del primer instituto de investigación de la antigüedad, al lado de la Biblioteca de Alejandría, incendiada 900 años después por el Califa Omar quien en acto justificatorio pronunció su célebre sofisma: "si los manuscritos de la biblioteca contradicen EI Corán, hay que quemarlos; si no los contradice, sobran, luego hay que quemarlos"

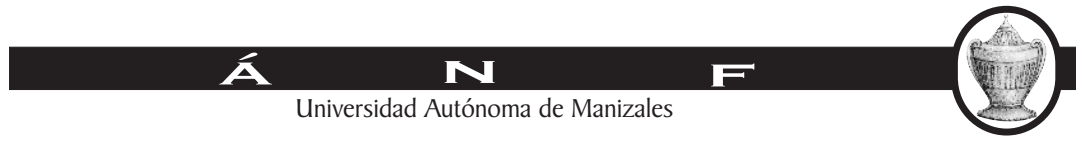


el hombre, cómo conoce, dónde vive, de dónde viene, para dónde va?

La resolución de estos interrogantes en la configuración de cuerpos teóricos sistemáticos, fue lo que permitió en la antigüedad la superación de la doxa (opinión o conocimiento vulgar) por la episteme (ciencia o conocimiento estructurado). A su vez, la resolución de cada uno de los interrogantes fue lo que dio origen a las diferentes ramas de la filosofía: la cosmología, la ontología, la gnoseología, la teogonía, la lógica, la física, entre otras. Para citar sólo un ejemplo, baste con recordar que la obra filosófica de Aristóteles está integrada por reflexiones lógicas, políticas, físicas, botánicas, retóricas, éticas y metafísicas. Recuérdese, además, que cuando se intenta dotar de contexto histórico a las ciencias reconocidas hoy como tales, siempre se termina invocando a pensadores presocráticos y a pensadores socráticos.

Que "la filosofía es la madre de todas las ciencias" es eslogan devenido en cliché, en esquema ocultante de una particularidad: el pensamiento antiguo y medieval (particularmente el de la baja Edad media), en tanto holístico, se definió por una actitud argumentativa y por una actitud hermenéutica. La alta Edad Media, en cambio, en tanto pensamiento de la realidad, sacralizó lo bíblico y lo impuso como fuente de toda verdad (ontológica, política, económica, jurídica, ética, estética, social, cosmológica, astronómica, matemática, física), redujo la filosofía a una sustentación teológica de los supremos misterios de la cristiandad, y erigió el teocentrismo como paradigma académico de las nacientes universidades y como principio de toda actuación humana.

En sentido estricto, la filosofía (antigua y medieval) no es la madre de las ciencias. Su aspiración teleológica de dotar de sentido la vida humana, partió de una concepción holística del mundo y de un método basado en la argumentación (deductiva) y en la interpretación (hermenéutica). Pero desestimó, quizá no por omisión, sino por convicción, la inducción y la prueba experimental. Por eso, siendo de naturalezas tan disímiles, no se le puede imputar ni maternidad ni paternidad al conocimiento filosófico prerenacentista con respecto a las ciencias originadas en el Renacimiento. 
Hay un aire de rebeldía como telón de fondo y quizá como síntoma de juventud- que puede explicar el tránsito de lo holístico a lo particular en el Renacimiento. La máxima de Bacon según la cual la verdad no hay que hallarla en el libro de La Biblia sino en el libro de la naturaleza, es indicativa de la rebelión frente al paradigma universitario de la época; simultáneamente, es premonitoria del paradigma de la modernidad: el antropocentrismo, la muerte de Dios, la decadencia de lo ecuménico, la irrupción del saber particular, la sacralización (paradójica en su momento) de la inducción.

En el intento de explicar la "megacrisis" del mundo actual, Max Neef argumenta que su causa es la tendencia moderna de la explicación del mundo por acción local y una tendencia cultural de mirar de soslayo y con desconfianza toda tentativa de comprensión del mundo como totalidad. El hecho es que, según el Nobel chileno,(3) la modernidad se dejó guiar no por un interés comprensivo de la realidad, sino por un interés de dominio, lo cual conduce, necesariamente, a una acción local, pues es imposible una acción dominante de totalidades.

Desde el Renacimiento, entonces, occidente inaugura una forma del conocimiento humano basado en un interés de dominio que condujo a la sectorización de la realidad. Esta forma del conocimiento, el científico, presenta unas características que determinan su separación definitiva de la filosofía: la predicción, la implementación de un método basado en la observación controlada de variables (experimentación), la formulación de resultados en lenguaje lógicomatemático, la traducción de resultados en tecnología y, finalmente, la definición de un objeto de estudio, es decir, la demarcación de un territorio exclusivo de acción local.

Queremos destacar, en este apartado que la referencia a La Ciencia, no desconoce su división en Formales, Sociales y Naturales, o la clasificación de la Escuela de Frankfut en Empírico Analíticas, Histórico Hermenéuticas y Crítico Sociales. El contexto al que se hace referencia demarca el significado de Ciencia como una forma del
3. Cfr. MAX NEEF, Manfred. De la esterilidad de la certeza a la fecundidad de la i n c e r t id u m b re. Transcripción de la conferencia sobre "el acto creativo", pronunciada a propósito del primer congreso internacional de creatividad. Bogotá, 1991.

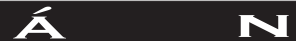

Universidad Autónoma de Manizales 
conocimiento de particularidades (no de totalidades), demarcación que es justificable en su momento por cuanto se trató de un proyecto cultural tendiente a establecer una ruptura con la tradición filosófica.

No obstante, si nos remontamos al Siglo XVIII, ese concepto de ciencia entra en crisis cuando se inaugura el debate acerca de si las características de las ciencias de lo particular subsumían a las nacientes ciencias dedicadas al estudio de fenómenos humanos (en sentido kantiano) o de si estas ciencias se configuraban por características particulares con respecto a aquéllas.

La diferenciación aludida entre conocimiento científico y conocimiento filosófico, así como la discusión de la noción de ciencia referida a la clasificación tradicional (formales y fácticas, naturales y sociales) o a los tres enfoques ("estilos", según Vasco), implica al mismo tiempo hacer referencia al significado que adopta la noción de teoría en cada caso.

En efecto, al conocimiento filosófico le es propia una noción de teoría como saber contemplativo, especulativo, como intelección, como principio de la práctica. Recuérdese que para los griegos(4) la diferencia entre teoría y praxis tiene un referente concreto: el espectáculo público que permite diferenciar el rol del espectador

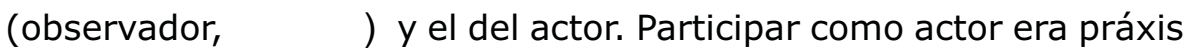
$(\bullet \bullet \bullet \bullet)$, en tanto que participar como observador era teoría $(\bullet \bullet \bullet \bullet)$ "Cuando el mirar, ver u observar se entendían 'mentalmente', el verbo •••• significaba 'considerar' o 'contemplar'; la theoría equivalía a contemplación. El sentido filosófico originario de teoría es el de contemplación, especulación, el resultado de la vida contemplativa o vida teórica." (5)

De esta manera, la relación teoría práctica era comprensiva y prescriptiva: la teoría se obtenía por vía hermenéutica y argumentativa, pero una vez difundida y apropiada se tornaba prescriptiva, al tiempo principio y fin de la praxis moral y de la praxis política. Nótese aquí como la teoría no se constituía ni se instituía
4 Cfr. FERRATER MORA, José. Diccionario filosófico. Tomo IV. Barcelona: Ariel. P.p $3.474-3.478$

5. Ibid. Pág. 3.475

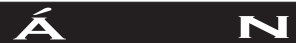

Universidad Autónoma de Manizales
F

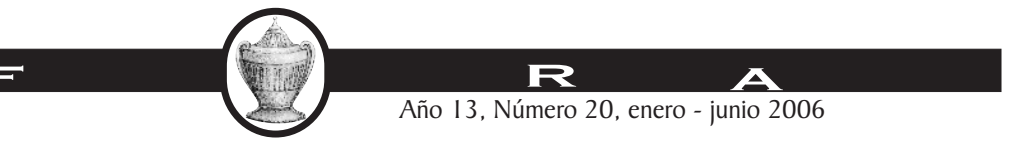


como un saber para el dominio de particularidades, sino más bien como una filosofía de vida, como un referente comprensivo y orientador de la vida humana: la teoría, pues, tenía una perspectiva teleológica.

Ahora bien, cuando se sucede la fragmentación de la filosofía con la aparición de la ciencia de lo particular, la teoría aquí también asume un rol específico. Al fragmentarse el ser en objetos de estudio, al imponerse la observación controlada de variables, al recurrirse a la matematización como condición explicativa de los objetos de estudio particulares, la teoría se instituye como una explicación estructurada y rigurosa del objeto de estudio, además fuente de hipótesis (por deducción) y referente de predicción y control de eventos relativos al mismo objeto de estudio.

La teoría, entonces, en este contexto tiene dos dimensiones: constituye un modelo de explicación del objeto de estudio, primera; $y$, segunda, halla su sello de validez en tanto sirve para anticipar y para dominar eventos relacionados con el objeto de estudio, es decir, el pragmatismo de la teoría es al tiempo su criterio de validez. Es esta la vía que permitiría, luego, estrechar los vínculos entre ciencia y tecnología.

Insistimos en que esta noción de teoría es relativa a la nueva ciencia, a la ciencia de corte baconiana, galileana y newtoniana. Buena parte de la tradición epistemológica heredada de la Ilustración y continuada por los defensores de las ciencias duras, se ha centrado en caracterizar las teorías como cuerpos sistemáticos en que se relacionan las leyes y en que se definen términos que luego adoptan el carácter de conceptos, todo esto con el propósito de establecer fronteras o puntos de diferenciación con respecto a otras formas del conocimiento humano, incluido el conocimiento por opinión o por sentido común. Dicho de otra manera, las teorías son el depósito de los tecnolectos de las ciencias, tecnolectos que se hacen cada vez más inexpugnables en tanto más especializaciones se generen en torno al objeto de estudio. 
La relación teoría-objeto de estudio es, pues, indubitable. Ahora bien, la manera como se entiende la teoría (el enfoque), como se construye y las características que posee, es lo que sí es objeto de discusión.

Por ejemplo, para Bunge la teoría "son sistemas conceptuales que intentan representar aspectos interrelacionados de sistemas reales."(6) Esta definición habría que inscribirla dentro del enfoque del realismo de las teorías, esto es, el enfoque según el cual la validez de las teorías está dada por su correspondencia con la realidad, la cual a su vez no depende ni de la existencia ni de la voluntad del científico; esta es la pretensión objetivista clásica del positivismo que pretende anular de la ciencia toda intervención subjetivista, todo sesgo valorativo.

Posición diferente a la anterior es la del antirrealismo o convencionalismo de las teorías, enfoque según el cual tanto los hechos como la realidad son construcciones humanas. Desde esta perspectiva, la concepción que un momento dado se tenga del mundo objetivo depende de la perspectiva teórica desde la que se aprecie. Así, el significado del mundo objetivo se hace diverso en tanto diversa es la acumulación teórica de los seres humanos en general y de los científicos en particular. Para Good, por ejemplo, la teoría "son relaciones entre hechos o el ordenamiento de los mismos en una forma en que tengan sentido."(7) No obstante, tanto las relaciones como los hechos mismos son construcciones humanas, son particularidades de los fenómenos. Si bien los hechos tienen correlatos empíricos, tienen un carácter objetivo, no son equivalentes: el hecho es construcción mental obtenida a base de la relación con fenómenos. Desde esta perspectiva, la pregunta por la validez o la invalidez de las teorías pierde pertinencia, en este contexto lo pertinente consiste en la utilidad de las teorías (como cuerpos sistemáticos generales) para la predicción y el dominio.
6 BUNGE, Mario. Citado por BISQUERRA, Rafael. Elementos de teoría científica. En: Módulo 1. Procesos de Construcción teórica. Maestría en Educación y Desarrollo h u m a no. Cinde Universidad de Manizales. Pág. 6

7. GOOD, William. Ciencia: teoría y hecho. Módulo 1. Op. Cit. Pág. 16

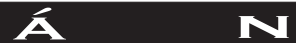

Universidad Autónoma de Manizales
$F$

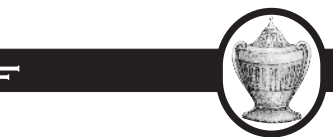


En tanto construcciones humanas, los hechos y las teorías están interrelacionadas. Para Good, los hechos ayudan a iniciar las teorías, contribuyen a su reformulación, son referentes de rechazo o de cambio de orientación. Y viceversa: las teorías permiten una clasificación de los hechos, son fuente de clasificación y sistematización de fenómenos, son el fundamento para la predicción y el control de los mismos y, además, son referente para identificar las claridades o los vacíos teóricos en una disciplina particular.(8)

Conceptos (entendidos en sentido kantiano como predicados de los fenómenos), leyes, axiomas (verdades evidentes y necesarias que no requieren demostración, según el postulado cartesiano), postulados (definidos por Bisquerra como proposiciones no evidentes que requieren demostración), teoremas (proposiciones que afirman una verdad demostrable según el mismo Bisquerra) y corolarios (entendidos como la consecuencia lógica de lo anterior al estilo de la lógica inferencial), así como variables y sus relaciones e hipótesis (supuestos predictivos), constituyen la estructura o los elementos estructurantes de las teorías.

Dos aspectos adicionales, a nuestro modo de ver, comportan las teorías desde el punto de vista de su utilidad: son determinantes para la construcción de objetos de estudio y son fuente para el ejercicio de las profesiones. En efecto, un objeto de estudio hay que pensarlo en dos dimensiones: una teórica y otra praxiológica. La primera está referida a la estructura teórica o a los conceptos de la o de las disciplinas desde las que se abordará el objeto de estudio. La segunda es la que permite configurar el campo de acción de la profesión que, siendo praxiológica, significa operacionalización del corpus teórico/conceptual disciplinar.

8 GOOD, William. Op. Cit. Pág. 17. 


\section{- SOBRE EL PAPEL DE LA TEORÍA EN LAS TRES FORMAS DE HACER CIENCIA}

Como antes se planteó, a partir del siglo XVIII se inicia una discusión relacionada con la reducción de la noción de ciencia natural a las ciencias sociales, lo cual implica desde luego la discusión sobre la noción de teoría. La disertación previa acerca de la configuración de las teorías científicas tiene validez relacionada específicamente con las ciencias de corte empírico analítico, dado su carácter explicativo, predictivo y su interés técnico de dominio.

Las ciencias de corte empírico analítico pretenden explicar los objetos de estudio, controlarlos, ejercer acción local sobre ellos y predecirlos. Para ello, han de apoyarse en cuerpos teóricos sistemáticos, generales, cuantitativos, nomológicos, a partir de los cuales se formulan hipótesis. Su carácter deductivo es evidente. Por ello, un proceso investigativo en este enfoque necesariamente debe partir del acumulado teórico relacionado con el objeto de estudio que pretende explicarse, tanto para identificar el problema de investigación como para responderlo previo al contacto con los referentes empíricos.

Aquí han de explicitarse las variables y sus relaciones (que le dan sentido a las hipótesis), han de establecerse los indicadores y sus sistemas de medición, esto es, han de definirse operacionalmente. Este procedimiento inicial configura el momento lógico de la investigación y le da sentido al momento metodológico (diseño de la investigación), a la captura de información y a su interpretación (momento lógico final). Si un proceso investigativo tiene por propósito encontrar respuestas teóricas a un problema, la teoría disponible precisa el problema, lo responde inicialmente, y también orienta el proceso metodológico, la toma de datos de la realidad y la respuesta final al problema, respuesta que opera nuevamente como teoría para confirmar la inicial, para enriquecerla o para falsarla.

Nótese que aquí ya no se está tomando como referencia la clasificación tradicional de las ciencias en formales, naturales y 
sociales. El enfoque empírico analítico se configura por el interés, más no por la tipología del objeto de estudio. Es decir, si el interés es explicar, controlar, predecir, ello puede hacerse tanto para responder a problemas relacionados con objetos de estudio de las ciencias naturales, como para objetos de estudio de las ciencias sociales.

Esta perspectiva, heredada de la Escuela de Francfurt, en cierta forma evita la generalización de la teoría para las ciencias naturales y para las ciencias sociales, y llama la atención sobre el peligro de positivizar las ciencias sociales y humanas. Pero también elimina las dicotomías y advierte sobre la necesidad de las investigaciones cuantitativas en las ciencias sociales, aunque advierte igualmente sobre su alcance.

Igualmente, otro aporte de la Escuela de Francfurt consiste en identificar otros dos tipos de intereses investigativos: el interés práctico y el interés emancipatorio. El interés práctico (praxiológico en la concepción de Vasco), consiste en comprender el contexto en que ocurren los hechos humanos, en reconstruir su sentido. Para poder comprender el contexto que le da sentido al obrar humano, es necesario partir de unas preguntas iniciales y de unos supuestos teóricos (ya no como hipótesis ni como falsación), sino como un medio a partir del cual se reconstruye un sentido.

Este punto de partida es el que se adopta en investigaciones históricas y etnográficas. Es decir, para reconstruir un sentido es preciso partir de unos conceptos iniciales, de unas categorías de análisis iniciales que enmarcan las preguntas y que orientan el momento metodológico de la investigación. Desde estos presupuestos se aborda la realidad para la toma de datos, no obstante, mientras la realidad en el enfoque empírico analítico aparece como fija, está dada al observador, los objetos de conocimiento históricos se manifiestan como texto, como lenguaje, como símbolo, por lo que en su indagación no es suficiente la observación, en cambio sí es necesaria la interpretación. 
En la propuesta de la Escuela de Francfurt, dado que para el interés comprensivo a las ciencias sociales no les es coherente el empleo de modelos causales, deben recurrir a la hermenéutica, a la "comprensión de sentido"; así, al contacto con los hechos humanos es que se reconfiguran las categorías de análisis, se precisan los presupuestos teóricos iniciales. La comprensión del sentido, en el momento lógico final, posibilita la reconstrucción teórica (no su falsación) que a su vez sirve como antecedente para proyectos de investigación que aborden objetos de conocimiento similares en otros contextos. La teoría, en este enfoque, no es universalizable, no tiene un valor explicativo y, por lo tanto, predictivo ni de control.

De manera similar al enfoque histórico hermenéutico, los procesos de investigación en la perspectiva crítico social parten de preguntas y de presupuestos teóricos iniciales por parte del investigador. La particularidad, en este caso, consiste en que el investigador ha vivido un proceso de inmersión en la comunidad, la conoce porque ha cohabitado $\mathrm{y}$, en este proceso, construye las preguntas $\mathrm{y}$ las categorías de análisis con la comunidad misma, deriva su saber, devela sus imaginarios, comprende sus particularidades.

El investigador crítico social revive la suerte de Sócrates, es una especie de partero de problemáticas y soluciones; pero, a diferencia de aquél, los alumbramientos no surgen exclusivamente del diálogo, sino también de su inmersión en el drama de los grupos humanos. Y también, a diferencia del filósofo, el investigador crítico social va dando cuenta de los alumbramientos en diarios de campo, en pautas de observación y somete a consideración de los grupos sus propias revelaciones.

Este participativo procedimiento "teórico-interrogativo-teórico" tiene un interés: la emancipación del grupo con el que se participa, esto es, la develación de las opresiones de diverso género para su liberación. Significa que, siendo este el interés, al investigador lo debe acompañar una teoría crítica de la sociedad, lo mueve un interés político, lo inspira la necesidad de la transformación social. 
La teoría crítica de la sociedad es un referente fundamental en este enfoque; también, los referentes teóricos de los grupos humanos que sufren las problemáticas y los antecedentes teóricos resultado de experiencias investigativas similares. El nexo "ciencia-sociedad" es evidente e indisoluble en este enfoque, dado que se traduce en incremento del capital teórico al servicio del mejoramiento de las condiciones de vida. 